\title{
For a Methodology to Implement Virtual Breeding Environments - A Case Study in the Mold and Die Sector in Brazil
}

\author{
Fabiano Baldo and Ricardo J. Rabelo \\ Department of Automation and Systems, Federal University of Santa Catarina, \\ PO Box 476, Zip Code 88040-970, \\ Florianópolis, Brazil \\ baldo@gsigma.ufsc.br, rabelo@das.ufsc.br
}

\begin{abstract}
This paper addresses the general problem of how classical cluster of industries can become a Virtual Breeding Environment (VBE). Using reference frameworks and models, a methodology to support this evolution is being conceived and its preliminary results are presented. This work tackles a cluster of moulds and dies industries from Brazil as the basis for the research and propositions. In the future, this methodology intends to serve as a concrete mean to systematize the implementation of a variety of VBEs.
\end{abstract}

Keywords: Virtual Breeding Environment, Virtual Enterprise, VBE implementation methodology.

\section{Introduction}

The importance of strategic alliances of diverse types has been pointed out by many authors as a way to increase companies' competitiveness [1]. Their benefits mainly depend on the type of alliances, varying from lobbying and sharing common services to dynamic composition of geographically dispersed companies to attend unique business opportunities [2].

In Brazil, since late 1990s several governmental and industry support institutions have promoted the formation of strategic alliances over the country, typically in the form of clusters and of the so-called local productive arrangements. Despite of the considerable benefits brought up with this initiative, it has been realized that such forms of alliances are limited by nature to face relevant requirements of nowadays economy, as volatility of economic barriers, harder global competition and higher innovation needs [3]. However, the problem is to identify which kind of alliance evolution they should pursuit and, once defined, how they should perform this? It is important to highlight that most of the companies are Small and Medium Enterprises (SMEs), which use to have a sort of restrictions to lead a deep study upon this.

There are several types of alliances. Virtual organization Breeding Environments (VBEs) [4] have arisen as a tremendously promising form of strategic alliance for 
reaching those requirements. A VBE can be generally defined as a long-term alliance of organizations aimed at offering the necessary conditions to support the rapid configuration of virtual organizations, strongly supported by ICT artifacts [5]. A number of references models, frameworks and prototypical software have been proposed to support such type of alliance, all of them with the potential to help companies in terms of providing theoretical foundations to evolve from e.g. a classical cluster to VBE. Nevertheless, while this helps answering the first part of the problem, this is not true at all for the second part: how to do that?

Actually, this is a complex issue as it embraces many technical (e.g. business processes, ICT and legal issues) and non-technical (e.g. organizations' cultures and working methods) perspectives, at several levels. In other words, there is not a simple and single answer to that. A first comprehensive attempt to answer this question, under a more technical-centric perspective, has been proposed by Romero et al. [5]. They have devised a methodology to create and to manage VBEs based on a reference framework for modeling collaborative networks $(\mathrm{CNs})$. In spite of its great importance, the methodology's steps are too abstract to be ready and easily applied to real cases of VBEs.

Trying to leave this methodology more concrete, this paper presents ongoing results of an applied, qualitative and combined case study and exploratory research, which is being carried out close to a cluster of mould and die companies in the south of Brazil. In general, it corresponds to an instantiation of Romero's work in order to better guide cluster's managers to form a VBE type of alliance.

This paper is organized as follows. Section 2 gives an overview on relevant VBE modeling frameworks. Section 3 depicts the mentioned Brazilian cluster, highlighting its main shortcomings. Section 4 presents the VBE model created to represent the cluster towards a VBE. Section 5 introduces the VBE methodology that is being developed. Conclusion and future work are presented at the end.

\section{VBE Modeling Frameworks}

Several works have approached the problem related to VBEs, generating a number of supporting models. From the scientific foundation point of view, the conceptual framework proposed by Hamided and Camarinha-Matos [4] has been considered as the most prominent basis for most of the works on VBEs.

In this framework, a VBE is not seen as a static multi-organizational entity, but rather as one which has its dynamics so having a life cycle: VBE initiation, recruiting, creation, operation, evolution, dissolution and metamorphosis phase. According to this framework, a set of activities have to be done within each phase. This includes the establishment and setup of a common base ICT infrastructure, VBE foundation, management of competencies, registration of new members, recording of past performance and collaboration in virtual enterprises/organizations, selection and reorganization of information and knowledge, inheritance management, governance, among many other activities.

In order to frame all this into a single and comprehensive model, VBE modeling frameworks are required. Next paragraphs provide a general overview about the most relevant works on VBE modeling. 
VERAM (Virtual Enterprise Reference Architecture and Methodology) [6] aimed at increasing the preparedness of entities involved in networks for efficient creation and operation of Virtual Enterprises (VE). VERAM facilitates the modeling process through the provision of guidelines on how to build the models and how to identify the common characteristics of VEs. Inspired in the CIMOSA enterprise framework [7], it includes a methodology (Virtual Enterprise Methodology - VEM) that describes how an organization should use the various components of the architectural framework during the VE engineering [8]. The VERAM model is divided in three main layers: 1) VE and networks Concepts; 2) VE Reference Architecture (VERA) [9] and; 3) VE Reference Architecture Components. Although not focused on VBE itself, it provided an important systematization of the process and organization of the framework's elements, which inspired other further works.

AmbianCE (Environment for creation of Virtual Enterprises) [3] is a framework developed to support the creation and operation of VBEs that are composed of SMEs. Adopting a bottom-up methodology, the cluster's companies have been firstly deeply analysed in order to design a more generic model of VBE. This cluster, called VIRFEBRAS, is now established in the city of Caxias do Sul, in the south of Brazil. . AmbianCE provides an environment where social capital, competence, use of ICT and knowledge reuse are the key elements for supporting a collaborative work. AmbianCE framework is used by means of three steps, namely AmbianCE Preparation, AmbianCE Structuring, and AmbianCE Acting. It strongly relies on knowledge management and benchmarking. These three steps are executed cyclically as a mechanism to guarantee the continuous VBE evolution.

Romero et al. [5] have created a methodology as a controlled process, addressing systematically a set of steps, supported by different mechanisms and methods required to establish and to characterize the management as well as to operate a VBE along its entire lifecycle. Based on the VBE reference framework developed by Afsarmanesh et al. [4], this methodology corresponds to an instantiation of ARCON modeling framework (see next paragraph) specifically for CNs of VBE type. The provided systematization is very useful as it not only transforms that framework in a sequence of more concrete steps, but also gives some guarantee that VBEs are to be created following more solid scientific theoretical foundations. Actually, this methodology corresponds to the so-called general perspective of ARCON, from which particularizations of CNs can be derived. This means that it is naturally too general to be ready applied when a real VBE is going to be created. Following ARCON, another perspective should be considered, which is the so-called specific one. This means that another methodology should be created to derive specific VBEs from this general methodology. This is exactly what this paper is about, and that will be explained in sections 4 and 5 .

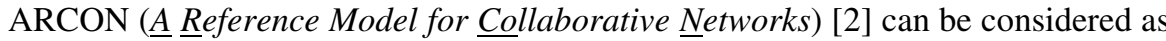
the most relevant modeling framework and reference model for CNs. Its basic rationale is the possibility to model generic and abstract representations for understanding the involved CN's entities and the relationship among them. ARCON intends to be used as the basis for deriving models for any manifestations of CNs. In very general terms, this is made applying three inter-related perspectives: 
- Life Cycle: It captures the evolution of $\mathrm{CN}$ and the diversity during their entire life cycle: creation, operation, evolution, dissolution and metamorphosis.

- Environment Characteristics: It focuses on capturing the CN environment characteristics and includes the internal elements ("Endogenous Elements") and the external interactions ("Exogenous Interactions").

- Model Intents: It is related to the different intents for the modeling of CN features and addresses three possible modeling stages: general, specific and implementation modeling.

\section{Mold and Die Cluster of Joinville City}

Due to the special importance SMEs have in Brazilian economy, several initiatives have been carried out by governmental and industry related institutions to promote their organization in the form of clusters to enhance their competitiveness in the internal and external markets.

Nuferj (Group of Moulds and Dies Industries of Joinville) is one of these clusters and represents the one that is going to be prepared to become a VBE. Joinville, a very industrialized medium-sized city placed in the State of Santa Catarina, in the south of Brazil, has about 450 moulds and dies industries and it is considered the largest pole of Latin America in this sector. Nuferj was founded in 1993 and it currently has 50 permanent members. Several members are competitors of the others. Automobile and household appliance companies are its main clients.

Moulds and dies are very unique parts. Each one uses to be very much complex to manufacture. Considering the increasing and extreme hard world-wide competition, Nuferj's members are more than ever exposed to - in a global market of US 20 billion - the competitiveness variables of cost, delivery date and quality. If investments on high-precision and high-speed CNC machines and on sophisticated CAD/CAM software have been enough to keep them competitive, this is no longer true nowadays. Other benefits of the cluster formation, such as the exchange of experiences and lobbying, are clearly not enough as well. That is the reason why Nuferj has been looking for an alternative model which allows it and its members to better and more effectively prepare themselves for the new reality.

Considering the extensive list of potential benefits that an alliance like VBE can add to Nuferj, they are investigating how this can be done. In this sense, the work presented in this paper corresponds to a contribution to this, identifying the required elements to propose an initial but more concrete methodology for a VBE creation, derived for the Nuferj case.

\section{Nuferj VBE Model}

From the methodological point of view, the derivation activity being done in this research assumes two essential premises: i) ARCON is comprehensive enough to effectively gathers the elements of work of real CNs and, ii) Romero's methodology is comprehensive enough to effectively gathers the Nuferj's elements of work. The 
underlying research hypothesis is that a VBE can be more properly created and further managed if more solid methodologies are used since the beginning of the process. This corresponds to a top-down approach, which tries to avoid the problems with the bottom-up approach, where the creation of VBEs is totally realized in a ad-hoc and empirical manners, as happened in the investigated cases [10].

Therefore, taking into account ARCON reference model and Romero's methodology, a NuFerj VBE Model has been created. This model classifies every element necessary to design the NuFerj VBE, which in turn will guide the work towards its implementation. According to ARCON, the NuFerj VBE model is a Specific Model that has been derived from the General Model specified by Romero's methodology. Table 1 presents the NuFerj VBE Specific Model, considering all the characteristics and elements involved in the Endogenous and Exogenous subspaces [11]. They include Structural elements (e.g. the actors that would be involved in the Nuferj VBE as well the existing roles), Componential (e.g. the type of resources necessary to operate the VBE), Functional (the business processes necessary to support its operation), Behavioral (elements that regulate its operation), Market (elements that are involved in the relationship between the VBE and the market), Support (the supporting entities involved and supporting services), Societal (elements that are involved in the relationship between the VBE and the society in general) and Constituency (actions mainly related with the attraction and selection of new members).

\section{Nuferj VBE Implementation Methodology}

Olave and Amato Neto [12] have discussed about the requirements of designing, implementing and sustaining clusters. They have highlighted three crucial aspects that are determinant for a cluster success: trust among partners, technical competence and excellence, and fluid use of ICT tools in their processes and collaborations. Therefore, creating a VBE requires a number of pre-requisites that should be incorporated in an implementation methodology. The problem is that none of those aspects are implemented at Nuferj. In this sense, this section introduces the current results of the VBE implementation methodology that is being developed looking at Nuferj's profile. This methodology aims at identifying all the activities necessary to cope with the main aspects modeled in the NuFerj VBE Model (Table 1). When totally finished, this methodology intends to serve as a concrete and systematized guideline to implement a variety of VBEs. As already mentioned, so far it is being customized for Nuferj.

Figure 1 shows the current status of the VBE implementation methodology, i.e. how the reference methodology is being derived to implement the Nuferj VBE. The methodology organization follows the VBE reference framework proposed by Hamideh and Camarinha-Matos [4], when identifies which steps should be done within the VBE life cycle. Next paragraphs detail the proposed methodology phases.

VBE Creation (Initiation \& Recruiting): this phase is mainly devoted to define the VBE mission and goals, its strategies in terms of marketing, political, economical issues, and the operating rules and duties. Although it may vary, a VBE usually begins with the so-called strategic members, whose selection can be accomplished by a sort of commission, depending on the local conditions. 


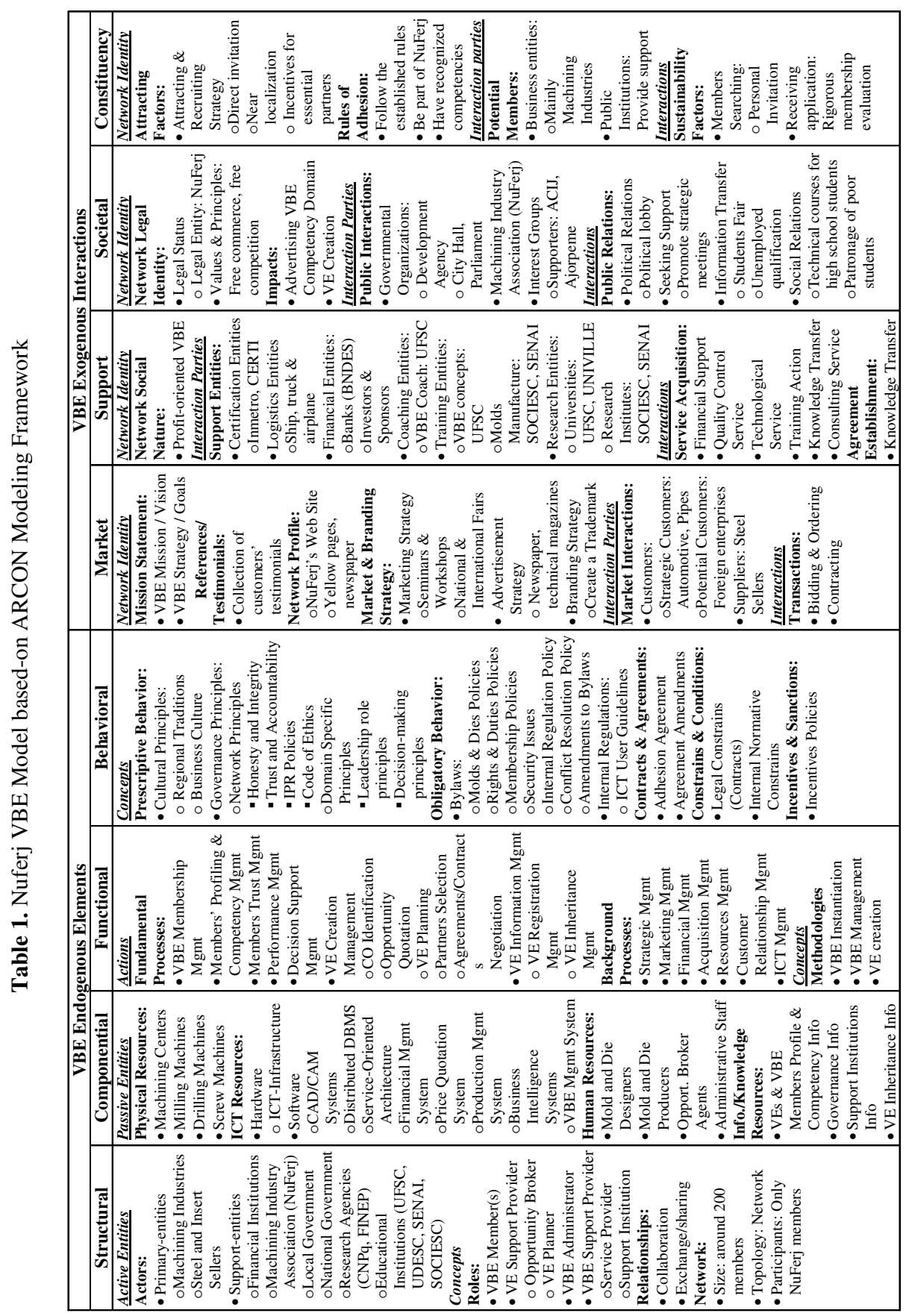


Business processes, ICT infrastructure and governance structure definitions complete the list of activities in this phase. Its legal establishment is also done.

VBE Creation (Foundation): in this phase the main activities are concerned to recruiting the VBE members (the companies themselves) and the VBE supporting institutions (e.g. educational, financial, R\&D institutions), whom a contract should be signed with. In the case of the VBE members, the envisaged competences should be firstly specified in order to ensure the right members will be invited. This does not mean that any of the mould and die company in Joinville region will be selected (although a VBE can comprise members from any region / country, only companies from Joinville are so far being considered). Every candidate should be empowered with basis knowledge on how to work in a/the VBE, which includes the aspect of trust (as most of the potential NuFerj VBE members are competitors). Once a given company is considered prepared to become a member, its roles are settled and its ICT infrastructure is prepared. Having all this prepared, the VBE can be launched.

VBE Operation: it is the phase which presents the largest number of activities. This phase is divided into two main subgroups: VBE Management and VE Creation. VBE management comprises the activities related to the full operation of the VBE and its main business processes. VE creation encompasses the activities devoted to the creation and initiation of new VEs once new collaboration opportunities are grasped (mostly) from the market. About the VBE management phase, in NurFerj the processes (and further systems) of Membership management and ICT infrastructure management should be firstly introduced. Profile and Competence Management activity comes as a sequence of the previous one. It includes the deployment of a system to register and to maintain the information related to the VBE members. A number of activities can start in parallel after this: Financial, Marketing, Performance, Acquisition, Trust, VE inheritance and Customer Relationship management. Acquisition management activity is critical for NuFerj as it is the one where the purchasing of raw material (mainly steel) is effectuated. This has a tremendous impact on the final cost of the mould or die, so it can represent winning or losing the business. Therefore, here is one concrete aspect where working collaboratively (i.e. buying together) can bring enormous business advantages.

About the VE creation phase, for NuFerj the Opportunity Identification management process/system is the first one to be deployed. This seems natural as it is responsible for brokering, fostering and gathering business opportunities to the VBE. The Opportunity Quotation management system/process is the next one to deploy. It is in charge of analyzing and making quotations for gathered clients' business opportunities. This is another critical activity in this sector as wrong quotations (not so abnormal regarding the complexity of this activity) can provoke very high financial losses. If the business opportunity is got, the necessary partners should be properly selected to compose the respective VE. The remaining activities are performed in sequence: Contract Negotiation management system/process (among the selected VE members, and between the VBE [or the company which got the business and that will usually act as the so-called VE coordinator] and the customer), VE Planning management system/process (to specify which partners will do what and when), VE Registration (to prepare the VBE's information repositories to store the information about the VE as well as to use this information for further inheritance management), and Launching management systems/processes (to coordinate the VE starting). 


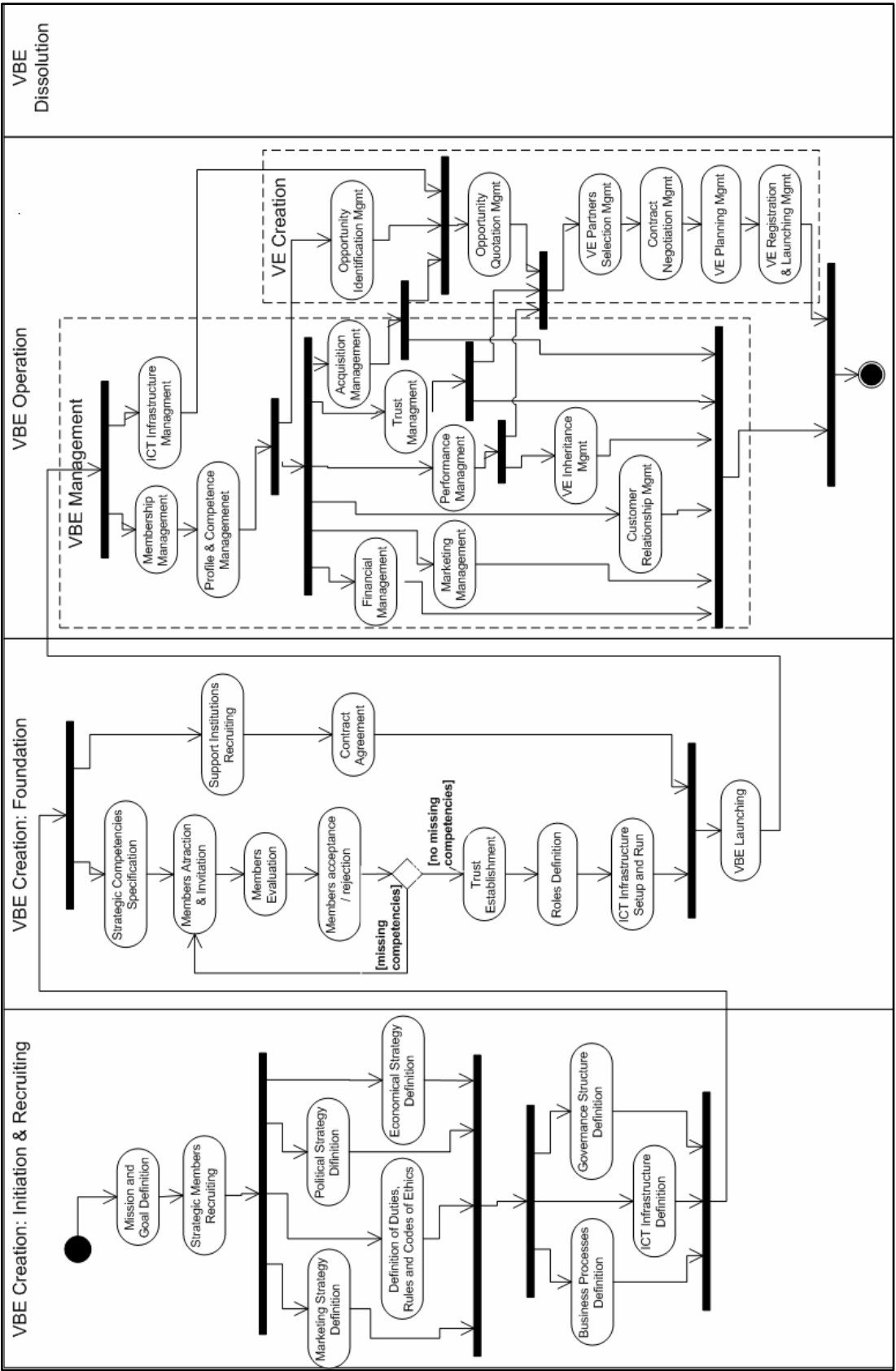

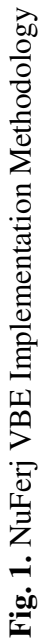


The methodology is essentially organized arranging the functional elements of the Nuferj VBE Model (Table 1) in a proper way considering the other model's elements along the several functional processes. For example, in the first stage of the VBE Creation the behavioral elements should be taking into account to e.g. set up the governance structure. In the VBE Creation / Foundation stage the structural elements of the model should be considered when selecting the VBE members and setting up their roles. In the VBE Operation stage, the market elements have to be considered in the marketing management business processes, and so forth.

As the current focus of the methodology is on how to create and launch a VBE, the phases of VBE dissolution and metamorphosis have not been addressed yet.

It is important to point out that Romero's methodology do not specify a strict sequence of actions, especially in VBE operation. This is natural as it is to be a reference methodology. As such, they only identify a set of general actions but that then need to be further adapted regarding the necessary particularities of the envisaged business environment / VBE. In this work, this has looked at Nuferj's environment. Other VBE can present different derivations.

\section{Conclusions}

This paper has presented the results of a methodology for implementing VBEs. It is a preliminary step towards a wider reference methodology for implementing VBEs. A cluster of mould and die industries has been used as a case study of this research.

A VBE model and methodology have been created, identifying the elements and processes required to create and launch a VBE. Although it is a bit generic, it provides a more detailed path when compared to other VBE methodologies, besides being applied to a real case.

It can be said that the developed methodology is compliant with the most relevant reference model for collaborative networks, which is ARCON modeling and reference framework. Although it is a very recent outcome and has not been deeply validated, its usage in practice showed feasible and comprehensive. In the interviews with Nuferj's representatives and in the methodology development, the necessity of all the identified elements has been confirmed.

The systematization provided by the methodology is very useful as it not only transforms a conceptual framework in a sequence of more concrete steps, but also it provides some guarantee that VBEs can be created following more solid scientific theoretical foundations, instead of purely ad-hoc recommendations.

Considering that the main purpose of this methodology is to implement VBEs, activities related to the dissolution and to the metamorphosis of such VBE have not been addressed yet.

As mentioned, the current version of the methodology has some limitations, which will be dealt with in the next phases of its development. Besides requiring a second stage of detailing in order to be even more concrete, the methodology does not cope yet with the temporality and complexity of the actions. For example, how long the establishment of trust among partners can take? How complex the mechanisms to empower partners can be, so that they can effectively be prepared to work in a VBE scenario? Other open points, which will be also focus in the continuation of this 
research, are related to the identification of the most critic actions (in terms of complexity and importance) as well as to the most sensible actions (in terms of VBE strategy and confidentiality against other competitors).

Only after this it is possible to go through the Implementation level of ARCON, instantiating all the models and tools (based on the devised and preliminary Specific level/model presented in this paper) to Nuferj and hence the creation of Nuferj VBE itself.

Acknowledgements. This work has been supported by Brazilian National Council for Scientific and Technological Development - CNPq. The authors thank Nuferj's managers, Mr. Alexandre Wanzuita and Mr. Christian Dihlmann, and the companies for the provided information.

\section{References}

1. Porter, M.E., Fuller, M.B.: Coalitions and global strategy. Competition in global industries 10, 315-343 (1986)

2. Camarinha-Matos, L.M., Afsarmanesh, H.: The ARCON Modeling Framework. In: Camarinha-Matos, L.M., Afsarmanesh, H. (eds.) Collaborative Networks: Reference Modeling, pp. 67-82. Springer, New York (2008)

3. Vallejos, R.V., Lima, C.P., Varvakis, G.: Towards the development of a framework to create a virtual organisation breeding environment in the mould and die sector. Journal of Intelligent Manufacturing 5, 587-597 (2007)

4. Afsarmanesh, H., Camarinha-Matos, L.M.: A Framework for Management of Virtual Organization Breeding Environments. In: Sixth IFIP Working Conference on Virtual Enterprises, pp. 35-48. Springer, Heidelberg (2005)

5. Romero, D., Galeano, N., Molina, A.: A Virtual Breeding Environment Reference Model and its Instantiation Methodology. In: Ninth IFIP Working Conference on Virtual Enterprises, pp. 15-24. Springer, Heidelberg (2008)

6. Zwegers, A., Tolle, M., Vesterager, J.: VERAM: Virtual Enterprise Reference Architecture and Methodology. VTT 1999, 17-38 (2003)

7. AMICE: CIMOSA - Open Systems Architecture for CIM, 2nd revised and extended edition. Research Report. ESPRIT Project (1993)

8. Zwegers, A., Hannus, M., Tølle, M.: Integration issues in virtual enterprises supported by an architectural framework. International IMS Project Forum - A common platform for cross-fertilization among IMS projects and promotion of the IMS program (2001)

9. Vesterager, J., Tolle, M., Bernus, P.: VERA: virtual enterprise reference architecture. VTT 1999, 39-52 (2003)

10. Galeano, N., Molina, A., Beeler, J., Monnier, F., et al.: VBE Pilot Demonstrators. In: Camarinha-Matos, L.M., Afsarmanesh, H., Ollus, M. (eds.) Methods and Tools for Collaborative Networked Organizations, pp. 405-430. Springer, New York (2008)

11. Camarinha-Matos, L.M., Afsarmanesh, H., Ermilova, E., Ferrada, F., et al.: ARCON Reference Models for Collaborative Networks. In: Camarinha-Matos, L.M., Afsarmanesh, H. (eds.) Collaborative Networks: Reference Modeling, pp. 83-112. Springer, New York (2008)

12. Olave, M.E.L., Amato Neto, J.: Redes de cooperação produtiva: uma estratégia de competitividade e sobrevivência para pequenas e médias empresas. Gestão \& Produção, pp. 289-318 (2001) 